\title{
Erratum: From single-particle excitations to sound waves in a box-trapped atomic Bose-Einstein condensate [Phys. Rev. A 99, 021601(R) (2019)]
}

\author{
Samuel J. Garratt, Christoph Eigen, Jinyi Zhang, Patrik Turzák, Raphael Lopes, \\ Robert P. Smith, Zoran Hadzibabic, and Nir Navon
}

(Received 15 July 2021; published 14 October 2021)

DOI: 10.1103/PhysRevA.104.049903

There is an error in the analytic $\omega^{(2)}$ expression (and consequently in Fig. 4 of the original paper). The text immediately following Eq. (17), "where $\mathcal{J} \approx 2.10$ is the radial factor in the overlap integral $I_{0011}^{\alpha}$ ", should read "where $\mathcal{J} \approx 3.15$ is the overlap integral $I_{0000}^{\alpha} "$. In the corrected Fig. 4 (shown below), we plot the corrected $\omega^{(2)}$ curve, which now coincides with the numerically calculated higher-order $\omega^{(i)}$ curves for small $g n /\left(\hbar \omega_{K}\right)$. This correction does not affect any of the conclusions of the article.

For clarity, note also that the factor $\eta_{j}$ in the exponent in Eq. (14) should read $\eta_{j}^{*}$, but for our $\hat{H}_{\text {kick }}$ and the modes of interest, $\eta_{j}$ can be chosen to be real. Similarly, the factor $Z_{j}$ in Eq. (16) should read $Z_{j}^{*}$.

We are grateful to Clara Tanghe and Karel van Acoleyen for bringing these points to our attention.

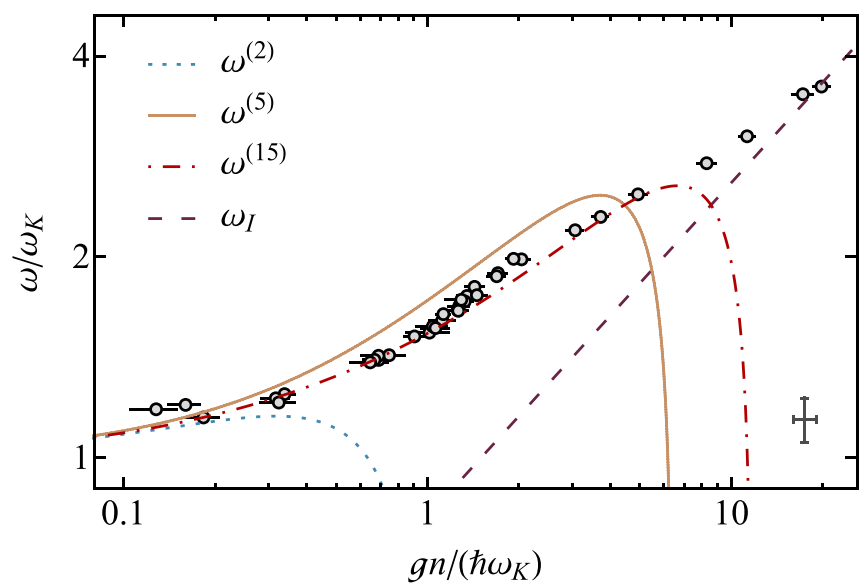

FIG. 4. Crossover from single-particle excitations to sound waves. We plot $\omega / \omega_{K}$ as a function of the interaction strength, now parametrized by $g n /\left(\hbar \omega_{K}\right)$. Here we assume $L=26 \mu \mathrm{m}$ and $R=16 \mu \mathrm{m}$ for calculations. The $x-y$ error bar in the bottom right corner indicates the fractional systematic uncertainties in the experimental data due to the uncertainties in the box dimensions. $\omega^{(2)}$ is determined using Bogoliubov theory within a truncated basis of just the two lowest-energy single-particle eigenstates of zero angular momentum (see text). This scheme fails even for relatively small $g n /\left(\hbar \omega_{K}\right)$, as it does not allow for the interaction-induced changes in the shape of the condensate or the excitation mode. $\omega^{(5)}$, based on a truncated basis of five single-particle eigenstates, is the minimal model that allows for the shape changes. This simple model already captures most of the crossover, and using progressively larger truncated bases does not qualitatively change the result, as shown by $\omega^{(15)}$, which is based on 15 single-particle eigenstates. $\omega_{I}$ is the sound-wave frequency, approached in the limit of large $g n /\left(\hbar \omega_{K}\right)$. 